\title{
Análisis en el reconocimiento, medición y revelación de ingresos por servicios para las pymes hoteleras del sector ecoturismo a partir de las NIIF para pymes
}

\section{Analysis in the recognition, measurement and disclosure of income from services for hotel SMEs in the ecotourism sector based on IFRS for SMES}

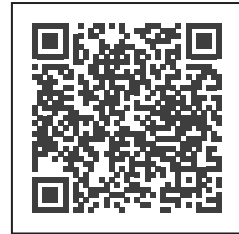

Palabras clave: contabilidad nacional; crecimiento económico; ecoturismo; industria hotelera; industria turística; ingreso; Normas Internacionales de Información Financiera (NIIF); pequeña empresa.

Artículo de Investigación Fecha de recepción: 12 de mayo de 2021

Fecha de aprobación: 13 de septiembre de 2021

Fecha de publicación: 22 de septiembre de 2021

Creative Commons Reconocimiento-NoComercial-SinObraDerivada 4.0 Internacional

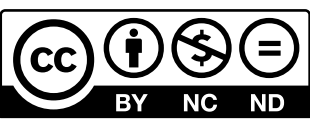

Oscar William Salinas Palacio';

Johanna Cristina Serrano Rincón²

\section{Resumen}

Problemática: tras la implementación del marco normativo de las NIIF para pymes en Colombia, las empresas hoteleras del sector ecoturismo han demostrado incrementos económicos relevantes, lo que ha aumentado su competitividad y productividad empresarial; sin embargo, son escasas las referencias bibliográficas que permiten analizar dichos efectos. Objetivos: se pretende analizar la forma que emplean estas empresas para reconocer, medir y revelar los ingresos de actividades ordinarias por los servicios de alojamiento de huéspedes. Materiales y métodos: se emplea una metodología dinámica que incluye la revisión documental del marco legal y normativo contable, repositorios académicos y el análisis de informes oficiales, aplicando una investigación exploratoria y explicativa

Estudiante de la Facultad de Ciencias Contables, Corporación Universitaria Remington, Bogotá, Colombia. Investigador del semillero SINCONT. Licenciado en Teología, Universidad de San Buenaventura, Bogotá. oscarwsalinas@gmail.com, ORCID: https://orcid.org/0000-0003-2008-2229

2 Docente de la Facultad de Ciencias Contables, Corporación Universitaria Remington, Bogotá, Colombia. Contadora pública de la Universidad Nacional de Colombia, Bogotá. Especialista en Revisoría Fiscal y Auditoría Externa y en Auditoría de Control Interno y Aseguramiento de la Universidad Libre. Candidata a magíster en Contabilidad de la Universidad Libre. johanna.serrano@uniremington.edu.co, ORCID: https://orcid.org/0000-0001-6889-9188 


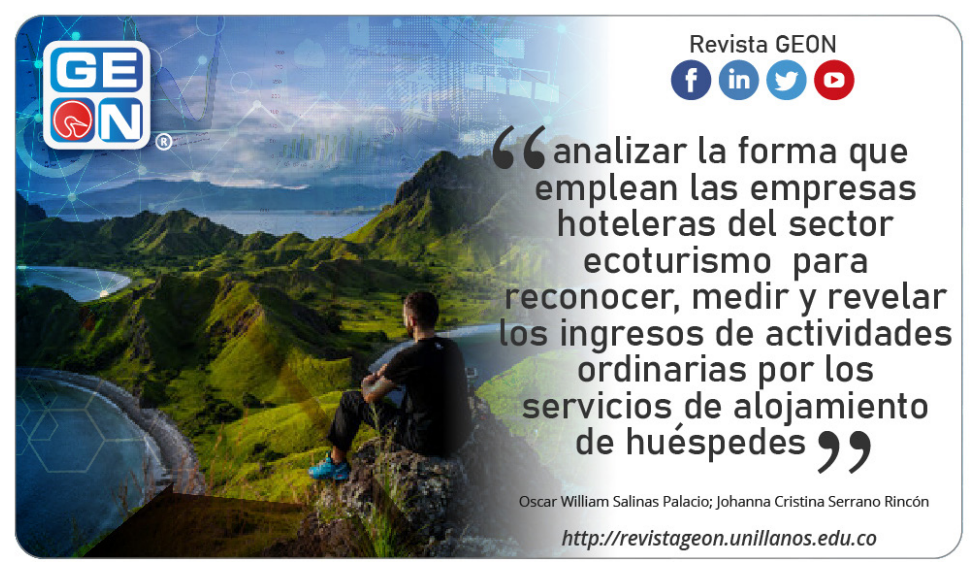

del marco técnico normativo vigente, mediante un enfoque mixto cuantitativo y cualitativo en la investigación. Resultados: los ingresos por servicios hoteleros se realizan conforme a los Decretos 2706 de 2012 y 3022 de 2013. Son reconocidos cuando se puede realizar una medición fiable y se traslada el control, los riesgos y las ventajas del bien o servicio al cliente. Su medición se fundamenta en el valor razonable aplicando el método de mercado activo. Discusión: la aplicación de la sección 23 de las NIIF para pymes no ha afectado el rendimiento económico de las pymes del sector ecoturismo; por el contrario, han generado mayor inversión extranjera y flujos de capital. Conclusiones: las empresas del grupo dos y tres aplican NIIF para pymes, las cuales pueden incorporar la sección 23 a sus políticas individuales. Contribución/originalidad: se espera formular una guía de implementación de la norma contable para el reconocimiento, medición y revelación de ingresos por servicios para las empresas hoteleras del sector ecoturismo que cumplan las exigencias para aplicar las NIIF del grupo dos y, en el caso de las microempresas del grupo tres, si quisieran incorporar la sección 23 a sus políticas individuales.

Salinas Palacio, O. W., \& Serrano Rincón, J. C. (2021). Análisis en el reconocimiento, medición y revelación de ingresos por servicios para las pymes hoteleras del sector ecoturismo a partir de las NIIF para pymes. Revista GEON (Gestión, Organizaciones Y Negocios), 8(2). https://doi. org/10.22579/23463910.498

Palabras clave: contabilidad nacional; crecimiento económico; ecoturismo; industria hotelera; industria turística; ingreso; Normas Internacionales de Información Financiera (NIIF); pequeña empresa. 
Códigos JEL: L83 Turismo; M41 Contabilidad; M48 Política pública y regulación; Q01 Desarrollo sostenible

\section{Abstract}

Problematic: After the implementation of the IFRS regulatory framework for SMEs in Colombia, hotel companies in the ecotourism sector have shown significant economic growth, which has increased its competitiveness and business productivity; however, there are few bibliographic references to allow the analysis of those effects. Objectives: the purpose is to analyze the methods used by these companies to recognize, measure and report revenues from ordinary activities for guest accommodation services. Materials and methods: the methodology used is a dynamic one that includes a documentary review of the legal and regulatory accounting framework, academic repositories, and the analysis of official reports, applying an exploratory and explanatory investigation of the current technical regulatory framework, using a mixed quantitative and qualitative research approach. Results: Revenues from hotel services are made according to Decrees 2706 of 2012 and 3022 of 2013 . The revenues are recognized when a credible measurement can be made and the control, risks, and benefits of the good or customer service are transferred. Its measurement is based on fair value applying the active market method. Discussion: The implementation of section 23 of IFRS for SMEs has not affected the economic performance of SMEs in the ecotourism sector; instead, it has generated greater foreign investment and capital flows. Conclusions: Companies of groups two and three apply IFRS for SMEs, which may incorporate section 23 into its individual policies. Contribution/originality: It is expected to formulate an implementation guide for the accounting standard for the recognition, measurement, and disclosure of service revenues for hotel companies in the ecotourism sector that meet the requirements to apply IFRS group two and, in the case of microenterprises in group three, if they wi- 
Cómo citar este artículo / Toreference this article:

Salinas Palacio, O. W., \& Serrano Rincón, J. C. (2021). Análisis en el reconocimiento, medición y revelación de ingresos por servicios para las pymes hoteleras del sector ecoturismo a partir de las NIIF para pymes. Revista GEON (Gestión, Organizaciones Y Negocios), 8(2). https://doi. org/10.22579/23463910.498 shed, they would be able to incorporate section 23 into their individual policies.

Keywords: Economic growth; Ecotourism; Hotel industry; Income; International Financial Reporting Standards (IFRS); National accounting; Small companies; Tourism industry.

JEL Codes: L83 Tourism; M41 Accounting; M48 Public policy and regulation; Q01 Sustainable development

\section{Introducción}

El presente estudio analiza la forma en que las empresas hoteleras del sector ecoturismo reconocen, miden y revelan los ingresos por servicios de alojamiento de huéspedes, bajo el marco normativo contable vigente en Colombia. Para cumplir esta tarea, la fundamentación teórica de la investigación se basa en la sección 23 de las Normas Internacionales de Información Financiera (NIIF) para pymes (ingresos de actividades ordinarias), aplicada a las empresas que se dedican al turismo ecológico. Así mismo, se basa en la consulta documental de repositorios académicos y en el análisis de los informes oficiales. Estos aportes en el ámbito profesional y científico generarán nuevas perspectivas de comprensión referentes a la implementación del marco normativo contable vigente para las empresas hoteleras del sector ecoturismo y permitirán conocer su impacto en el reconocimiento de los ingresos por servicios hoteleros. Del mismo modo, en materia de investigación, permitirá obtener estadísticas y mediciones del impacto económico que han generado las pymes en la economía con relación a la implementación del marco normativo contable, buscando explicar el fenómeno actual de las empresas dedicadas al ecoturismo, para fundamentar una tesis apropiada y acorde a las propuestas referenciales.

Por lo anterior, en la propuesta de investigación se analizan las dificultades de pasar de la norma local a la norma internacional, intentando resolver la siguiente incógnita: ¿de qué forma las pymes hoteleras del sector ecoturismo reconocen, miden y revelan sus ingresos por servicios a partir de las NIIF? Esta pregunta da la apertura para establecer otras preguntas de investigación sobre las cuales se fundamenta el presente documento: ¿cómo aplican el marco normativo vigente en Colombia las empresas hoteleras del sector ecoturismo para reconocer, medir y revelar sus ingresos?, ¿cuál es el criterio de valoración más adecuado establecido en el marco normativo contable colombiano para medir y revelar dichos ingresos?, ¿qué estudios académicos existen 
para comprender el objeto de estudio de la presente investigación? Las respuestas a estos interrogantes permiten analizar la forma que emplean las empresas hoteleras del sector ecoturismo para reconocer, medir y revelar los ingresos de sus actividades ordinarias por los servicios de alojamiento de huéspedes.

Los resultados de la investigación evidencian que las empresas que pertenecen al turismo ecológico obtienen diversos ingresos provenientes del portafolio de servicios que ofrecen a sus clientes y, además, incorporan la sección 23 de las NIIF para pymes en sus políticas contables para reconocer, medir y revelar estos ingresos, utilizando el valor razonable como base de medición.

\section{Contexto teórico}

Marco técnico normativo empleado por las pymes hoteleras para reconocer, medir y revelar sus principales fuentes de ingresos

El tema objeto de la investigación puede ser ubicado dentro de las siguientes teorías e información existentes:

\section{NIIF para pequeñas y medianas empresas (pymes)}

Según Fierro-Martínez y Fierro-Celis (2015, p. 22), las NIIF para pymes son un conjunto de normas contables y financieras expedidas por el International Accounting Standard Board (IASB), con el fin de emplearse en los estados financieros de información general para entidades que no están obligadas a reportar públicamente sus balances. Botero et al. (2018, p.
134) indican que el marco normativo que se aplica a las pequeñas y medianas empresas se encuentra reglamentado por los Decretos 3022 de 2013 y 2420 de 2015, los cuales están conformados por 35 secciones de NIIF para pymes.

Las empresas hoteleras del sector ecoturismo aplican el marco técnico normativo establecido en la sección 23 de las NIIF para pymes y reglamentado en Colombia mediante el Decreto Único Reglamentario (DUR) 3022 de 2013 (DUR 2420 de 2015, Anexo 2). No obstante, existen algunos hoteles que son microempresas y aplican la contabilidad simplificada establecida en los Decretos 2706 de 2012, 2106 de 2016 y 2132 de 2016, tal como lo informa Bolaños (2018, p. 9) en su artículo de investigación.

De acuerdo con Vanegas (2006, p. 5) y con el informe de Viajala (2020), las principales fuentes de ingresos de las empresas hoteleras, que pertenecen al sector ecoturismo, están relacionadas con el cobro de servicios de alojamiento de huéspedes y con las unidades generadoras de efectivo (UGE), que incluyen alquiler de ecohabitaciones, miradores, huerta orgánica, restaurante, lavandería, spa, parqueadero, avistamiento de aves, tours (en carros, motos y caminatas), senderos ecológicos, deportes ecoaventura y cocina vegetariana orgánica, entre otros.

\section{Reconocimiento de ingresos por actividades ordinarias}

Según la Fundación IASC (2009), en la sección 23 de las NIIF para pymes el 
reconocimiento de ingresos por actividades ordinarias consiste en la incorporación de un registro en el estado de resultados, con alta probabilidad de obtener beneficios económicos futuros relacionados con ingresos por actividades ordinarias, pero que puedan medirse con fiabilidad. Este ingreso puede estar representado por la venta de bienes, la prestación de servicios, contratos de construcción, intereses, regalías y dividendos.

\section{Valor razonable}

Siguiendo la definición proporcionada por Hoogervorst (2015) y las Normas Internacionales de Información Financiera (2011), existen algunas partidas de los estados financieros para las entidades que hacen parte del sector hotelero, las cuales se miden a valor razonable, como los ingresos que devenga por la prestación de servicios de alojamiento, que actualiza al precio del mercado con fines de negociación, al igual que todas aquellas unidades generadoras de efectivo que proporcionan ingresos adicionales para su operación. La Fundación IASC (2009), en la sección 23 de las NIIF para pymes, define al valor razonable como el precio por el que un activo puede intercambiarse, un pasivo liquidarse o un instrumento que hace parte del patrimonio ser comercializado con los interesados sin generar ninguna dependencia entre sí, lo que, en otras palabras, puede interpretarse como el valor por el cual podría ser vendido un activo o pagarse al trasladar un pasivo en una avenencia mercantil para la fecha en que se realice la medición respectiva.

\section{Costo histórico}

De acuerdo con el portal español de Normas Internacionales de Información Financiera NIC-NIIF (2015), las empresas del sector ecoturismo utilizan el costo histórico para depreciar la propiedad, planta y equipo, reflejando el consumo de la vida útil del activo $y$, en este proceso, la gerencia de la empresa aplica cierta subjetividad para estimar el valor en uso de su deterioro conforme a los flujos futuros de efectivo. Es necesario aclarar que dichos activos son usados por la compañía para ejercer su actividad económica, razón por la cual no requiere aplicar el método de valor razonable.

\section{Ecoturismo}

Hace referencia al turismo sostenible y especializado, el cual se debe realizar en las "áreas con atractivo natural especial [...] buscando la recreación, el esparcimiento y la educación del visitante a través de la observación, el estudio y la admiración de los valores naturales y de los espacios culturales" (Rodríguez et al. 2015, p. 31). De acuerdo con Costas (2018), el ecoturismo debe tener en cuenta tres pilares fundamentales: prácticas ambientales apropiadas, preservación de las herencias culturales y naturales y beneficios económicos, tanto locales como nacionales, que involucren a las comunidades de la región. En lo referente a las prácticas ambientales fundamentadas en la responsabilidad social empresarial (RSE), se ha observado que las empresas hoteleras basan sus esfuerzos en desarrollar su actividad económica a partir 
de prácticas sostenibles tendientes a preservar y cuidar el medio ambiente. Acorde con Peña et al. (2018, p. 177), la RSE debe implementarse en la industria hotelera, dada la relación que tiene con los ecosistemas y la población de base, pues genera un impacto negativo en la naturaleza y en la comunidad local, desarrollando actividades que implican un cierto grado de contaminación auditiva, deterioro del aire, el aumento de deshechos y basuras, así como la irrupción del ciclo de vida en algunos ecosistemas.

Gracias a la aplicación de los anteriores criterios de negocio y proyección turística, los informes y estadísticas proporcionados por el Departamento Administrativo Nacional de Estadística ([DANE], 2020, p. 10), el Ministerio de Comercio Industria y Turismo ([MinCIT], 2020, p. 38) y la Organización Mundial del Turismo ([OMT], 2019) evidencian el auge de las empresas hoteleras del sector ecoturismo, quienes han presentado un importante crecimiento del 10,6\% en sus ingresos durante los últimos años, según informe del DANE (2020, p. 9), destacándose sobre otros sectores de la economía. No obstante, tras la reciente emergencia sanitaria generada debido a la COVID-19, este sector de la economía se ha visto gravemente afectado.

\section{Empresas hoteleras}

Según lo afirman los autores Rondi et al. (2020, p. 21), la actividad hotelera es el engranaje principal dentro de la industria turística, la cual ha tenido un gran auge durante los últimos años, pasando a ser el principal actor para el comercio nacional e internacional. De acuerdo con Vanegas (2006, p. 5) y Viajala (2020), entre las principales fuentes de ingresos por servicios hoteleros se incluyen otras actividades concordantes con el objeto socioeconómico de dichas empresas, que les permiten obtener recursos económicos, incrementando de manera significativa sus finanzas.

\section{Caracterización del sector hotelero en Colombia}

Para lograr altos estándares de calidad a nivel internacional, a partir del año 2016 las empresas en Colombia fueron clasificadas por la Dirección de Impuestos y Aduanas Nacionales (DIAN) en tres grandes grupos para la implementación de las NIIF, como lo afirman Franco (2018, p. 22) y Bolaños (2018, p. 5):

- Grupo 1: entidades hoteleras que realizan cotización en la bolsa de valores y venden acciones al público, captan o manejan recursos del público, cuentan con activos mayores a 30.000 SMMLV, tienen más de 200 empleados y están asociadas con compañías extranjeras que aplican las NIIF. De acuerdo con el Decreto 2784 de 2012, las empresas que pertenecen al grupo 1 deben aplicar las NIIF plenas.

- Grupo 2: empresas hoteleras que no realizan cotización en la bolsa de valores y no manejan recursos del público, sus activos totales están comprendidos desde 500 hasta 30.000 SMMLV, tienen entre 
11 y 200 empleados y sus ingresos brutos al año son mayores de 6000 SMMLV. A las empresas que hacen parte de este grupo se les denomina pyme (empresa pequeña y mediana), las cuales llegan al 99\% del total de la industria hotelera colombiana. Debido a esto, el gobierno nacional instituyó una versión simplificada de las NIIF plenas, Ilamada NIIF para pymes, regulada por el DUR 3022 de 2013.

- Grupo 3: empresas minoristas, conformadas por personas naturales y jurídicas, las cuales no hacen parte del régimen simplificado en cuanto al impuesto sobre las ventas, cuyos ingresos brutos no superan los 3500 SMMLV, tienen un total de activos por valor de 500 SMMLV y cuentan máximo con 10 empleados. Las microempresas que pertenecen a este grupo deben aplicar las NIF, que es un tipo de contabilidad simplificada, con el fin de estandarizar la presentación y revelación de los estados financieros. Esto está reglamentado mediante el Decreto 2706 de 2012.

Según los informes estadísticos del DANE (2020, p. 10) y del MinCIT (2020, p. 38), la mayor parte de la industria hotelera colombiana, que desarroIla actividades de ecoturismo, se encuentra dentro del grupo 2 y 3 en implementación de NIIF y su margen porcentual de ocupación hotelera en 2019 fue del 57,8\%, 15 puntos porcentuales mayor en comparación con el año 2018, constituyendo la cifra más alta de la historia en Colombia.
No obstante, tras el inicio de la pandemia ocasionada por la COVID-19 a nivel mundial, las empresas que se dedican al turismo fueron gravemente afectadas.

\section{Normatividad contable expedida por el gobierno nacional}

Las empresas hoteleras que pertenecen al sector ecoturismo reconocen, miden y revelan los ingresos por prestación de servicios de alojamiento de huéspedes a partir de los lineamientos definidos en la sección 23 de las NIIF para pymes, regulada por los Decretos 3022 de 2013 y 2420 de 2015, expedidos por el gobierno de Colombia (Botero et al., 2018, p. 134). En función de esta norma contable, las empresas hoteleras reconocen sus ingresos conforme lo prescribe dicha norma, al incorporar una partida en el estado de resultados, en la cual es posible que la empresa genere en el futuro un beneficio económico relacionado con los registros contables por ingresos de actividades ordinarias y que se mida de manera fiable acorde al precio de intercambio en un mercado activo. Por ello, la Fundación IASC (2009) define el valor razonable como el criterio de medición para dichos ingresos. La revelación de estas partidas contables está definida por la misma norma, la cual establece que se deben revelar las políticas contables utilizadas para el reconocimiento y medición de los ingresos, así como el valor de cada clase de ingresos registrados en el período sobre el que se informa. También, Barbosa et al. (2018, p. 61) recomiendan a las 
empresas contar con la asesoría de un profesional en Contaduría y con la ayuda de sistemas de información tecnológicos para la preparación de las notas a los estados financieros que, de forma específica, deben realizarse de manera narrativa. Así mismo, se debe informar sobre las políticas contables significativas aplicadas en cada registro contable.

En cuanto a la emisión de normas contables en el mundo, existen dos organismos independientes que pertenecen al sector privado: la Junta de Normas de Contabilidad Financiera (FASB) y la Junta de Normas Internacionales de Contabilidad (IASB). La FASB fue instaurada en el año 1973 y sustituyó a la Comisión de Principios del Área Contable, fundada en 1959, cuyo principal propósito consistía en la creación de normas y pronunciamientos contables para servir como guía en la presentación de reportes e información financiera en Estados Unidos (NICNIIF, s.f.). Por su parte, la IASB reemplazó al Comité de Normas Internacionales de Contabilidad (IASC), el cual era el encargado de emitir las Normas Internacionales de Contabilidad NIC - IAS. Es así como dese el año 2001 el IASB se ha ocupado en desarrollar y aprobar las Normas Internacionales de Información Financiera (NIIF), siendo supervisado por la Fundación del Comité de Normas Internacionales de Contabilidad (IASCF).

Según lo afirman Botero et al. (2018, p. 136), en Colombia se reglamentaron las NIIF a partir de la Ley 1314 de 2009, bajo los Decretos 2784 de 2012, 2420 de 2015 y 2496 de 2015 para el grupo 1, cuyos integrantes son emisores de valores, están conforma- dos por entidades públicas y aplican las NIIF plenas. En los DUR 3022 de 2015 y 2420 de 2015 se reguló el grupo 2, representado por empresas medianas y pequeñas no emisoras de valores ni públicas, las cuales aplican las NIIF para pymes. Mediante los Decretos 2706 de 2012, 2106 de 2016 y 2132 de 2016 se reglamentó el grupo 3, conformado por microempresas, a las que se les autorizó la emisión de estados financieros y revelaciones de forma abreviada. Estas deben aplicar la contabilidad simplificada.

En la tabla 1 se relaciona el marco legal colombiano, conformado por la Ley 1314 de 2009 y los decretos que regulan la implementación de las NIIF en Colombia para las empresas que pertenecen a los grupos 1, 2 y 3, respectivamente.

Según Borrero y Ortiz (2016, p. 64), a partir de la Ley de Convergencia 1314 de 2009, Colombia inició la regulación de las NIIF, siendo actualmente una realidad en el país. De acuerdo con Fierro-Martínez y Fierro-Celis (2015, p. 24), dicho proceso se llevó a cabo a partir del dinamismo que experimentaron las economías a nivel mundial, haciendo que muchos países adopten y adapten estándares internacionales como las NIIF plenas y también las NIIF para pymes.

\section{NIIF para pymes en el sector hotelero: reconocimiento, medición y revelación en los estados financieros de las empresas hoteleras}

La Fundación IASC(2009), en la sección 23 de las NIIF para pymes, determina 
Tabla 1.Marco legal de Colombia en el proceso de implementación de las Normas Internacionales de Información Financiera

\begin{tabular}{ccc}
$\begin{array}{c}\text { Grupo 1 } \\
\text { NIIF plenas }\end{array}$ & $\begin{array}{c}\text { Grupo 2 } \\
\text { NIIF para pymes }\end{array}$ & $\begin{array}{c}\text { Grupo 3 } \\
\text { Microempresas }\end{array}$ \\
\hline Ley 1314 de 2009 & Ley 1314 de 2009 & Ley 1314 de 2009 \\
\hline Decreto 2784 de 2012 & & Decreto 2706 de 2012 \\
\hline Decreto 1851 de 2013 & Decreto 3022 de 2013 & Decreto 3019 de 2013 \\
\hline Decreto 3023 de 2013 & & \\
\hline Decreto 3024 de 2014 & Decreto 2129 de 2014 & \\
\hline Decreto 2267 de 2014 & & \\
\hline Decreto 2615 de 2014 & & Decreto Único Reglamentario \\
\hline Decreto Único Reglamentario & Decreto Único Reglamentario de 2015 \\
2420 de 2015 & 2420 de 2015 & Anexo DR 2706 de 2012 \\
\hline Anexo DR 2615 de 2014 y DR & Anexo DR 3022 de 2013 y DR & 2131 de 2016 \\
2131 de 2016 & Decreto Reglamentario 2496 & Decreto Reglamentario 2496 \\
de 2015
\end{tabular}

Fuente: adaptado de Botero et al. (2018, p. 139).

que el reconocimiento de los ingresos por actividades ordinarias, procedentes de los servicios prestados por estas entidades, debe efectuarse conforme al índice de cumplimiento de la transacción, finalizando sobre el período que se está informando. Dicho índice debe ser medido de manera fiable, al igual que los costos invertidos, para completar la transacción. Si no se cumple este lineamiento, en los ingresos deben reconocerse únicamente los gastos que se estimen recuperables. Del mismo modo, la sección 23 de las NIIF para pymes establece que el reconocimiento de los ingresos por actividades ordinarias debe hacerse en el momento en que el vendedor transfiera al comprador todos los riesgos y ventajas inherentes del bien o servicio que adquiere, en cuyo caso el vendedor no puede retener el control seguro sobre ellos. Así mismo, esta norma aclara que el reconocimiento debe realizarse con fiabilidad. Esta sección también determina que la medición de ingresos por actividades ordinarias en la empresa deberá efectuarse "al valor razonable de la contraprestación recibida o por recibir, dicha medición debe tener en cuenta los descuentos comerciales, descuentos por pronto pago y rebajas por volumen de ventas" (párrafo 23.3).

En cuanto al pago con moneda extranjera por una contraprestación anticipada, el Comité de Interpretaciones de Información Financiera (ClNIIF 22), conforme al IFRS Fundation (2016), establece que la tasa de cambio a tener en cuenta en dichos registros debe medirse en el momento de realizarse el cobro efectivo del anticipo, es decir, aquella que se determina en el reconocimiento inicial del activo, gasto o ingreso conexo. De hecho, el Estatuto Tributario Colombiano en 
los artículos 27 y 28, modificado por la Ley 1819 de 2016, indica que el reconocimiento y la medición de los ingresos se generan cuando haya lugar a un aumento en los activos o un decrecimiento de los pasivos, cuya medición se pueda establecer con fiabilidad y cuando ocurran en el período gravable.

\section{Materiales y métodos}

Con el objetivo de identificar el marco normativo contable que se debe aplicar a las entidades que se dedican al turismo ecológico, el cual incluye las NIIF y algunos decretos que permiten cumplir el marco legal, la presente investigación se enfoca en la revisión literariay documental de los postulados teóricos a nivel normativo contable de varios autores y tiene en cuenta los informes estadísticos de organismos nacionales, para analizar la manera en que las empresas hoteleras del sector ecoturismo reconocen, miden y revelan los ingresos por servicios de alojamiento, en cumplimiento de su objeto socioeconómico. Para ello, se realiza el estudio de las líneas de negocio del ecoturismo y su registro contable, el cual deriva en políticas individuales de reconocimiento y valoración de ingresos, considerando, de esta manera, la importancia que tienen las NIIF para pymes en relación con el incremento de la competitividad y la productividad de las empresas hoteleras del sector ecoturismo.

\section{Tipo de estudio}

La investigación es de carácter exploratoria y explicativa, dado que el tema de análisis ha sido poco estudiado y se requiere mayor profundidad para explicar la temática propuesta. El tipo de estudio es explicativo, debido a que se detalla la aplicación del marco normativo técnico vigente en las empresas hoteleras del sector ecoturismo para comprender su impacto en el incremento de ingresos observados.

\section{Métodos}

Para el presente estudio se realizó la revisión documental del marco legal y normativo contable, repositorios académicos e informes oficiales, tanto del gobierno nacional como de organismos internacionales, para analizar la forma en que las empresas hoteleras del sector ecoturismo reconocen, miden y revelan los ingresos de servicios de alojamiento en cumplimiento de su objeto socioeconómico, sobre el marco normativo contable vigente en Colombia. Incluso, mediante el análisis cualitativo y cuantitativo de los datos estadísticos hallados en la revisión documental, se estableció la relación con el tema de investigación, logrando revelar la forma de aplicar el marco normativo contable vigente y su incidencia en el reconocimiento de los ingresos por servicios hoteleros, realizando así un importante aporte en el ámbito académico, ya que no se cuenta con mucha producción científica en el país sobre el sector ecoturismo.

A continuación, se presentan algunas posturas sobre las investigaciones relacionadas con el proceso de implementación del marco normativo contable de las NIIF para pequeñas y medianas empresas que pertenecen 
al sector ecoturismo. Para ello, se consultaron algunas bases de datos de repositorios de varias universidades.

Chiriboga (2013, p. 16), en su artículo llamado Aplicación de la sección 23: ingresos de actividades ordinarias en una empresa pyme del turismo receptivo, indica que las actividades ordinarias de la empresa deben reconocerse en la medida en que sea factible que los beneficios económicos futuros se dirijan hacia la entidad y estos deben ser medidos de manera fiable. Del mismo modo, el autor afirma que la entidad deberá revelar las políticas contables y los métodos manejados para el reconocimiento de dichos ingresos.

Fierro-Martínez y Fierro-Celis (2015, p. 114), en su libro Contabilidad general con enfoque NIIF para pymes, desarrollan una postura relacional y estrecha entre la contabilidad y los negocios empresariales, formulando el ciclo contable, los informes y los fundamentos teóricos en función de las necesidades organizacionales de las diversas entidades económicas.

En el artículo Pymes implementación de normas de información financiera internacional (grupo 2), describen el proceso de implementación de las NIIF en Colombia desde el punto de vista experiencial de las pymes hoteleras del Caribe colombiano, indicando la desinformación generada sobre el tema (Borrero y Ortiz, 2016) sumado a la falta de orientación por parte de los organismos responsables de la preparación y presentación de la información financiera (Del Franco Blanco et al, 2019).
Bolaños (2018, p. 6) establece la relación entre el costo y el beneficio en la adopción de las NIIF por parte de las pequeñas y medianas empresas en Colombia, mostrando las consecuencias a nivel económico y empresarial, pero exaltando el desempeño realizado por las mipymes, las cuales se han destacado a nivel competitivo y productivo, convirtiéndose en el motor de la economía del país. Costas (2018), en su artículo Colombia tiene gran potencial para desarrollar turismo sostenible, propone tres pilares fundamentales para el sostenimiento económico, organizacional y competitivo del turismo colombiano: en primera instancia, propone las prácticas ambientales ecológicas; en segunda instancia, la conservación de las tradiciones culturales locales; y en última instancia, los beneficios bilaterales tanto para los locales como para los nacionales.

La investigación realizada por Rondi et al. (2020, p. 22), en su artículo titulado Reconocimiento y medición de ingresos en la actividad hotelera, hace un análisis de los procedimientos que debe llevar a cabo el profesional contable al evaluar las estimaciones contables de los servicios de alojamiento que efectúan estas empresas, indicando que se deben reconocer los ingresos en los períodos que efectivamente se prestan.

\section{Análisis estadístico}

El enfoque de la investigación es mixto porque combina el análisis cuantitativo y cualitativo de los datos consultados, dado que se recolecta información estadística con medición 
numérica para analizarla y establecer su relación con el tema de investigación, revelando la forma de aplicar el marco normativo contable vigente y su impacto en el reconocimiento de los ingresos por servicios hoteleros. Siguiendo este enfoque en la investigación, se pudo comprobar que el número de establecimientos de alojamiento y hospedaje legalmente constituidos en Colombia es de 27.640 para enero de 2020, según Burgueño (2020) y estadísticas nacionales del Registro Nacional de Turismo ([RNT], 2021). De estos, el 37,62\% pertenece al sector hotelero de ecoturismo. Como resultado del análisis en la información consultada, en la tabla 2 se ilustran las empresas activas de turismo de naturaleza, clasificadas por su tamaño, que prestan servicios de alojamiento a huéspedes.

Del mismo modo, siguiendo las mediciones estadísticas del DANE (2020, p. 10) y los resultados obtenidos en las investigaciones desarrolladas por Borrero y Ortiz (2016, p. 132), Bolaños (2018, p. 5), y Botero et al. (2018, p. 138), se evidencia que las empresas hoteleras que hacen parte del turismo de naturaleza aplican el marco normativo contable vigente en las proporciones que muestra la figura 1, en donde es evidente que la mayoría de empresas implementan las NIIF para pymes con el fin de reconocer, medir y revelar sus ingresos por servicios hoteleros. Dichas empresas están representadas por microempresas (mipymes), así como por pequeñas y medianas empresas (pymes), quienes han decidido establecer en sus políticas la sección 23 de las NIIF para pymes. El porcentaje total de empresas que se acogen a este marco normativo es del $62 \%$, seguido por un 37\% que aplica la contabilidad simplificada (NIF) y un 1\% de empresas grandes que pertenecen al grupo 1, las cuales aplican las NIIF plenas.

Tabla 2. Empresas hoteleras en Colombia, año 2021

\begin{tabular}{lccc|c} 
Clasificación de empresas & \multicolumn{2}{|c|}{ Ecoturismo } & \multicolumn{2}{c}{ Otros sectores económicos } \\
\cline { 2 - 5 } \multicolumn{1}{c}{ hoteleras por tamaño } & Activas & Porcentaje & Activas & Porcentaje \\
\hline Grupo 1: grandes & 104 & $0,37 \%$ & 172 & $0,62 \%$ \\
\hline Grupo 2: pequeñas y medianas & 9048 & $32,72 \%$ & 2068 & $7,48 \%$ \\
\hline Grupo 3: microempresas & 1248 & $4,51 \%$ & 15.000 & $54,29 \%$ \\
Total & 10.400 & $37,6 \%$ & 17.240 & $62,4 \%$ \\
\hline
\end{tabular}

Fuente: elaboración propia. 
Figura 1. Implementación del marco normativo contable en empresas hoteleras del sector ecoturismo.

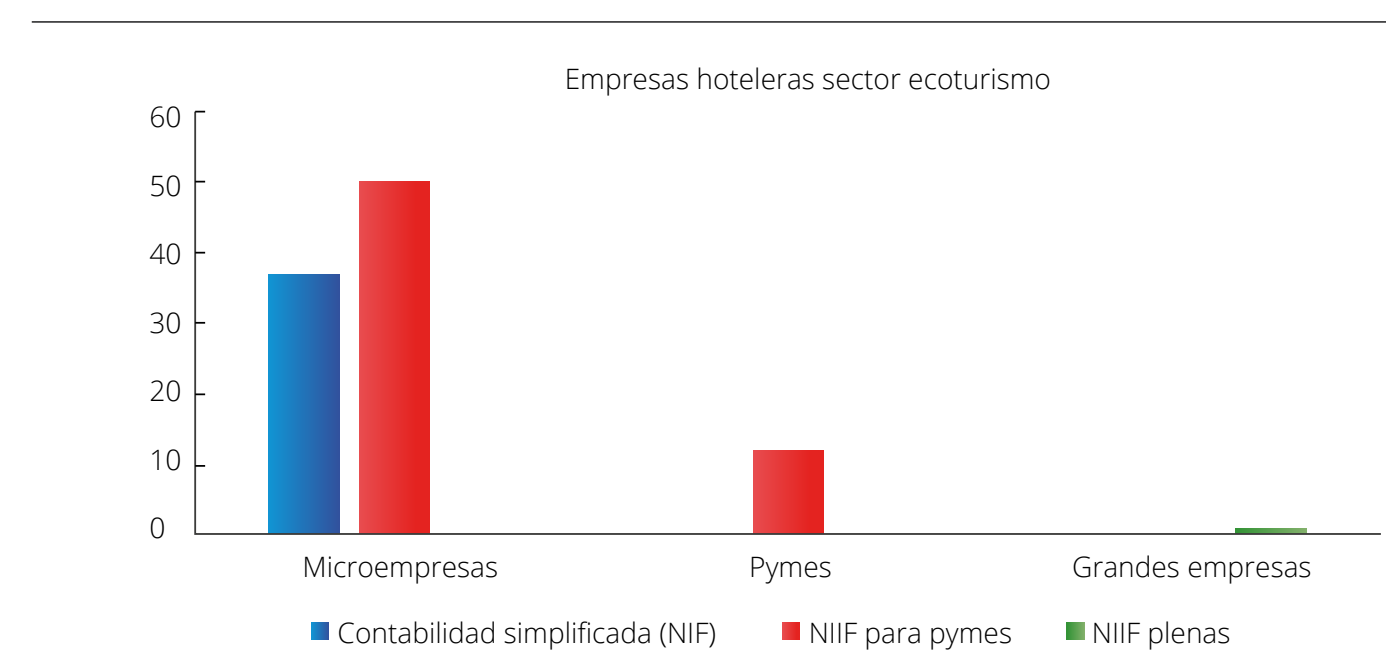

Fuente: elaboración propia.

\section{Resultados y discusión}

El desarrollo de la investigación arrojó algunos resultados importantes que se relacionan en la tabla 3, entre ellos, el análisis de las debilidades, oportunidades y fortalezas, así como la identificación de las amenazas que pueden perturbar la actividad del turismo ecológico y, por ende, afectar los ingresos, en especial, los del sector hotelero, con lo cual se pretende fortalecer las empresas que brindan estos servicios y proyectar resultados óptimos para el desarrollo de su actividad económica.

En materia normativa y legal, se identificó que las empresas hoteleras del sector ecoturismo, que pertenecen al segundo grupo en adopción de NIIF, implementan el marco técnico normativo establecido en la sección 23 de las NIIF para pymes y reglamentado en Colombia mediante el DUR 3022 de 2013 (DUR 2420 de 2015, Anexo
2). Sin embargo, algunos hoteles son microempresas que aplican la contabilidad simplificada establecida en los Decretos 2706 de 2012, 2106 de 2016 y 2132 de 2016. Estas empresas han impulsado el turismo de naturaleza a nivel nacional con ahínco, sensibilizando sobre la importancia de cuidar y preservar el medio ambiente. De hecho, en la figura 2 se muestra el escalafón por departamentos, en el cual es notable el grado de influencia que ejerce el ecoturismo a nivel nacional.

Según los informes del DANE (2020, p. 3) y del MinCIT (2019, p. 25), los ingresos hoteleros aumentaron significativamente entre el año 2010 y 2019; sin embargo, con ocasión de la pandemia generada por la COVID-19, estos se han reducido considerablemente en el año 2020 y en lo corrido del año 2021 (DANE, 2021, p. 13), decayendo el margen porcentual y afectando su aporte al producto interno bruto (PIB). 
Uscar William Salinas Palacia; Jahanna Cristina Serrana Rincán

Tabla 3. Análisis DOFA ecoturismo

Debilidades

Falta de capacitación oportuna y consecuente de los empleados que laboran en la prestación de servicios hoteleros.

Guías turísticos sin los conocimientos necesarios para brindar un servicio acorde al portafolio hotelero.

En cuanto a las lenguas nativas y extranjeras, es evidente la falta de preparación y certificación.

Infraestructura hotelera ineficiente y poco congruente con los estándares internacionales de turismo ecológico.

Presupuestos escasos y sin proyección financiera, los cuales se preparan de manera ineficiente, solo con el objetivo de atender la demanda actual de servicios.

Precarias condiciones en la construcción de carreteras que comunican las diferentes zonas turísticas.

Poco interés por parte de la administración para optar por certificaciones de calidad que permitan alcanzar altos estándares de conformidad entre los clientes.

\section{Oportunidades}

Integración de la población nativa para trabajar de forma conjunta en el logro de los objetivos financieros y organizacionales de la región.

Preservación de los parques naturales, humedales y demás hábitats que conforman la plataforma natural e infraestructura necesaria para el desarrollo social y cultural del país.

Conformación de equipos de trabajo, tanto nacionales como extranjeros, que incentiven el desarrollo social, económico y cultural, promoviendo el turismo de naturaleza. Destinación de recursos por parte del Estado para las regiones que implementen el turismo ecológico como parte estratégica de su portafolio de servicios turísticos.

Desarrollo de programas sociales de sensibilización, con énfasis en la atención oportuna de incidentes naturales ocasionados por el ser humano, buscando mitigar los riesgos ambientales.
Fortalezas

En el territorio nacional existen más de cincuenta zonas de protección natural, las cuales aportan una alta biodiversidad y provisión de recursos naturales.

Localizaciones protegidas por convenciones internacionales, las cuales buscan elevar el equilibrio natural del país.

Sitios estratégicos de preservación de fauna y flora.

Colombia cuenta con aproximadamente doscientas cincuenta zonas de reserva natural y turística, en donde abundan diferentes hábitats que permiten la coexistencia de especies.

Colombia se destaca porque a nivel avícola supera en número la cantidad de ejemplares de cualquier país del mundo.

\section{Amenazas}

El conflicto armado y los grupos de delincuencia común, los cuales no permiten el desplazamiento libre y seguro de los turistas. Naciones con mejores ofertas turísticas, mayores infraestructuras naturales, protección y garantía para el libre desplazamiento de los turistas, mejores fondos de inversión financiera para los proyectos naturales, entre otros aspectos.

Falta de conciencia de preservación del medio ambiente por parte de las poblaciones nativas y visitantes, lo cual puede afectar gravemente los ecosistemas en proyecciones futuras.

Recortes de presupuestos nacionales para el desarrollo de programas que incentiven el turismo ecológico.

Nota: La tabla 3 representa el análisis DOFA de los productos y servicios del turismo de naturaleza para Colombia (TNC). Fuente: adaptado de T\&L Turism Leisure \& Sports y Avia Export (2012, p. 515). 
Figura 2. Desarrollo del ecoturismo en los 32 departamentos de Colombia.

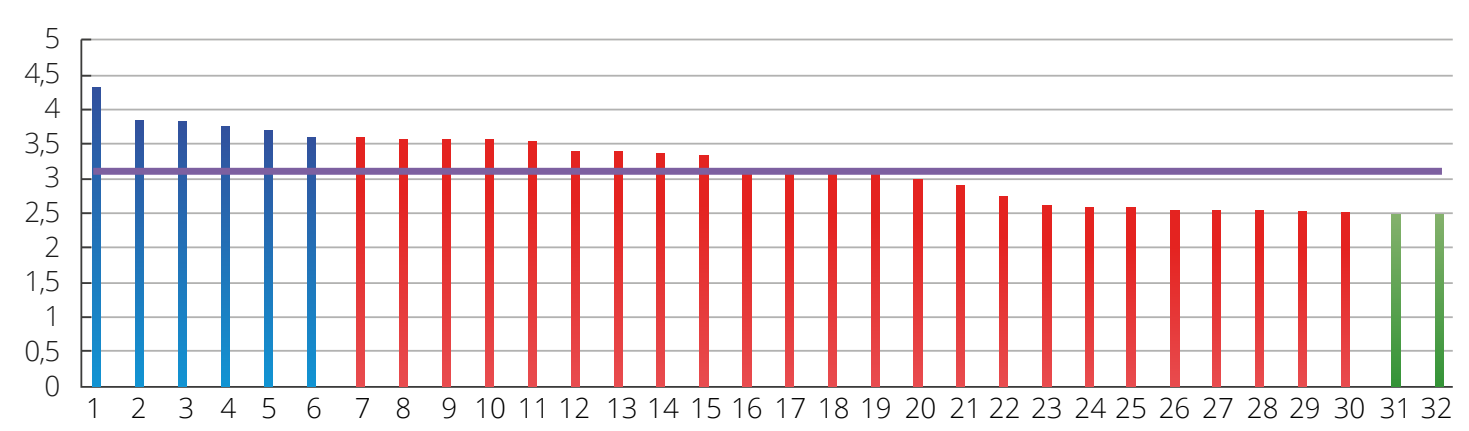

Grado excelente / bueno Grado aceptable Grado regular — Media país

$\begin{array}{lllll}\text { 1. Magdalena } & \text { 7. Chocó } & \text { 14. Huila } & \text { 20. Cesar } & \text { 26. Guaviare } \\ \text { 2. Bogotá y } & \text { 8. Caldas } & \text { 15. Meta } & \text { 21. Casanare } & \text { 27. Caquetá } \\ \text { Cundinamarca } & \text { 9. Risaralda } & \text { 16. Cauca } & \text { 22. Putumayo } & \text { 28. Vichada } \\ \text { 3. Antioquia } & \text { 10. Boyacá } & \text { 17. San Andrés } & \text { 23. Córdoba } & \text { 29. Guainía } \\ \text { 4. Valle del Cauca } & \text { 11. Bolívar } & \text { y Providencia } & \text { 24. Norte de } & \text { 30. Sucre } \\ \text { 5. Quindío } & \text { 12. Guajira } & \text { 18. Tolima } & \text { Santander } & \text { 31. Vaupés } \\ \text { 6. Amazonas } & \text { 13. Santander } & \text { 19. Nariño } & \text { 25. Atlántico } & \text { 32. Arauca }\end{array}$

Fuente: adaptado de T\&L Turism Leisure \& Sports y Avia Export (2012, p. 514).

Es importante resaltar que los hoteles que se dedican al turismo ecológico obtienen su ingreso principal por el servicio de alojamiento de huéspedes, como se puede observar en la figura 3. No obstante, en el análisis realizado por Vanegas (2012, p. 5) y Viajala (2020), dentro de las líneas de negocio existen unidades generadoras de efectivo (UGE) que producen ingresos de forma independiente para las empresas hoteleras, tales como alquiler de ecohabitaciones, miradores, huerta orgánica, restaurante, lavandería, spa, parqueadero, avistamiento de aves, tours (en carros, motos y caminatas), senderos ecológicos, deportes ecoaventura, cocina vegetariana orgánica, entre otros. Es por esta razón que Salazar (2011, p. 224) y Rondi et al. (2020, p. 22) concluyen que el reconocimiento de ingresos bajo las NIIF se aplica cuando el hotel puede realizar una medición fiable y transfiere al cliente el control, los riesgos y las ventajas concernientes al bien o servicio.

La base de medición de los ingresos que utilizan las empresas hoteleras se fundamenta en el valor razonable, como lo especifica Hoogervorst (2015), aplicando el método de mercado activo y la revelación de ingresos por servicios hoteleros, lo cual se realiza acorde con la sección 23 de las NIIF para pymes (Fundación IASC, 2009), en la que se establece el deber de revelar las políticas contables utilizadas para el reconocimiento y medi- 
ción de los ingresos, así como el valor y la clase de ingresos reconocidos en el período sobre el que se informa (Moncayo, 2015).

Finalmente, es necesario destacar que la implementación de las NIIF para pymes facilita el conocimiento de la situación financiera de la entidad, así como su rendimiento económico y permite proyectar sus flujos de efectivo, siendo esta información importante para la toma de decisiones, tal como se explica en el módulo 23 emitido por la Fundación IASC (2009). Aunque algunas empresas no cumplen con las exigencias para aplicar las NIIF grupo 2 y son microempresas del grupo 3, pueden incorporar la sección 23 de las NIIF para pymes a sus políticas individuales, considerando la presente investigación como referencia.

Figura 3. Fuentes de ingresos del ecoturismo y unidades generadoras de efectivo.

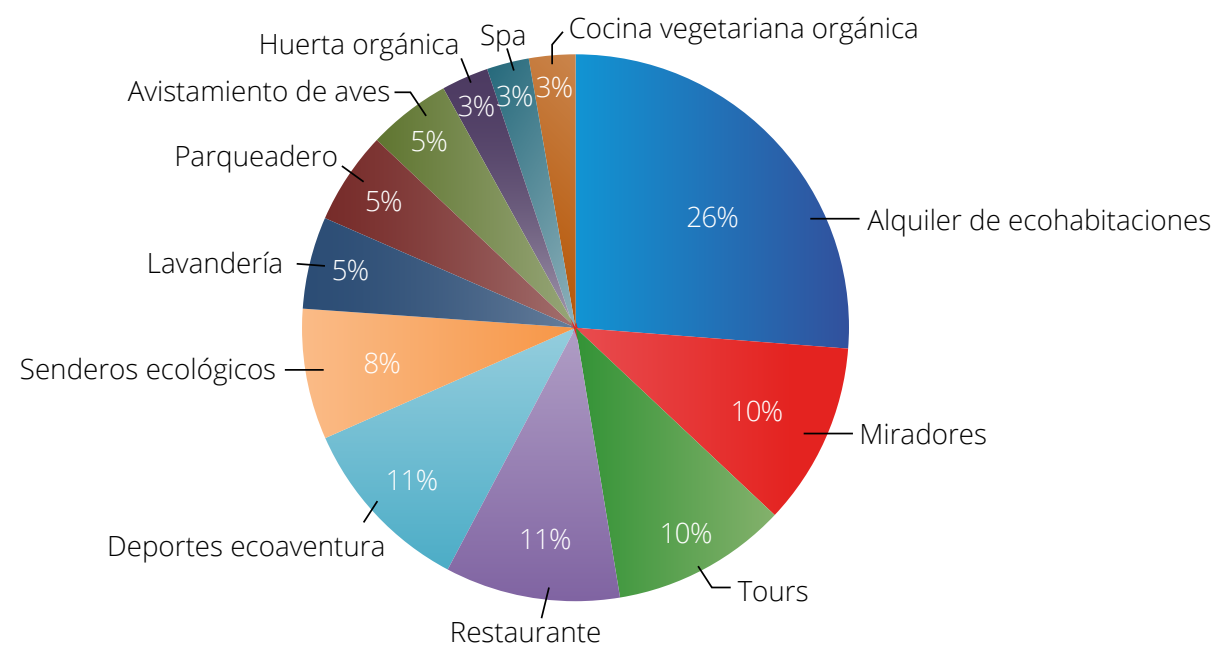

Fuente: elaboración propia a partir de Vanegas (2012, p. 5) y Viajala (2020).

\section{Conclusiones}

Según los informes y estadísticas emitidos por organismos oficiales, se comprobó que los ingresos por servicios hoteleros de ecoturismo tuvieron un incremento significativo desde el año 2010 al 2019, aumentando así su competitividad y productividad empresarial. Sin embargo, debido a la pandemia generada por la COVID-19, estos ingresos se han reducido con- siderablemente en los años 2020 y 2021, decayendo el margen porcentual en cifras y afectando su aporte al PIB. Ante este hecho y con el fin de analizar la forma que emplean las empresas hoteleras de ecoturismo para reconocer, medir y revelar sus ingresos, la presente investigación se fundamentó en la revisión documental del marco legal y normativo contable, los repositorios académicos y el análisis de los informes oficiales, 
evidenciando que la implementación de la sección 23 de las NIIF para pymes establece los lineamientos para el reconocimiento, medición y revelación de los ingresos ordinarios que desarrollan las pequeñas y medianas empresas dedicadas al turismo ecológico. Del mismo modo, se observó que la aplicación de este marco normativo no ha afectado el rendimiento económico de las pymes del sector ecoturismo, por el contrario, ha generado mayor inversión extranjera y flujos de capital, aumentando el acceso al sector financiero por la homogeneidad de reconocimiento, medición y revelación de hechos económicos. Incluso, en el ámbito contable, el proceso de implementación de las NIIF para pymes en Colombia ha favorecido el registro de las operaciones para estas entidades, brindando ventajas significativas en cuanto a la medición fiable de los ingresos por servicios hoteleros y unidades generadoras de efectivo, lo que ha permitido aumentar así su competitividad y productividad empresarial.

Por lo anterior, y debido a la metodología empleada en la investigación, es posible inferir la objetividad y transparencia con la cual se desarrolló el presente estudio, permitiendo alcanzar conclusiones reveladoras que comprueban la aplicación del marco técnico normativo formulado en la sección 23 de las NIIF para pymes y reglamentado en Colombia mediante los DUR 3022 de 2013 y 2420 de 2015 Anexo 2 por parte de las pymes hoteleras del sector ecoturismo. No obstante, también se evidenció que algunos hoteles son microempresas, quienes aplican la contabilidad simplificada establecida en el DUR 2706 de 2012. Así mismo, se comprobó que estas entidades obtienen su principal fuente de ingreso por alojamiento de huéspedes y también por unidades generadoras de efectivo, las cuales incorporan en el desarrollo de su actividad económica. Del mismo modo, se comprobó que el reconocimiento de ingresos por actividades ordinarias bajo las NIIF se aplica cuando el hotel puede realizar una medición fiable y transfiere el control, los riesgos y las ventajas concernientes al bien o servicio al cliente. Esta medición se fundamenta en el valor razonable aplicando el método de mercado activo. Finalmente, se evidenció que la revelación de ingresos por servicios hoteleros, en las mipymes y en las pymes, se fundamenta en los estándares establecidos para la presentación y revelación de los estados financieros, reglamentado mediante los Decretos 2706 de 2012 y 3022 de 2013 en Colombia, los cuales formulan las directrices para la revelación de las políticas contables utilizadas para el reconocimiento y medición de los ingresos, así como el valor y la clase de ingresos por servicios hoteleros reconocidos en el período que se informa.

Con esta investigación se espera formular una guía para el reconocimiento, medición y revelación de ingresos por servicios para las empresas hoteleras del sector ecoturismo que cumplan los requisitos para aplicar las NIIF del grupo 2. En el caso de las mipymes del grupo 3, si quisieran incorporar la sección 23 a sus políticas individuales, esta investigación servirá como 
referencia para la conformación de un modelo ejemplar de implementación de la norma contable, dado que el desarrollo científico del trabajo documental es consistente y aumenta la comprensión temática de la investigación, debido a que actualmente hay poco trabajo académico relacionado con el sector ecoturismo.

\section{Agradecimientos}

Son muchos los benefactores que han contribuido en la presente investigación. A todos ellos quiero agradecer su noble labor desinteresada, en especial a la docente Johanna Cristina Serrano por su apoyo incondicional durante mi formación académica y profesional; al coordinador académico Wilber García por su efectiva gestión; a la Universidad Remington por permitirme cursar mis estudios universitarios con calidad y profesionalismo; a mis padres, hermanos y familia por su entereza, cariño, amor y comprensión; a Dios a quien dedico mis mayores logros.

\section{Contribuciones de autoría}

El autor principal Oscar William Salinas Palacio realizó la revisión documental, el análisis, la redacción y presentación del artículo de reflexión. Así mismo, la docente Johanna Cristina Serrano contribuyó en la asesoría temática y metodológica para la conformación del presente trabajo de investigación.

\section{Conflictos de interés}

Tanto el autor principal, Oscar William Salinas Palacio, como la docente Jo- hanna Cristina Serrano, declaramos que no existe ninguna relación, condición, posición o circunstancia que afecte la objetividad del manuscrito, pues su elaboración pretende analizar de manera objetiva la forma que emplean las empresas hoteleras del sector ecoturismo para reconocer, medir y revelar los ingresos por servicios a partir de las NIIF para pymes.

\section{Financiamiento}

La presente investigación fue financiada con los recursos propios de los autores, producto de su esfuerzo y dedicación investigativa en el área contable y financiera.

\section{Material suplementario}

No existe material suplementario para el presente artículo de reflexión, por lo cual sugerimos que sean revisadas las referencias bibliográficas para ampliación del tema objeto de estudio.

\section{Referencias}

Barbosa-Martínez, D. J., Chagualo-Galindo, M. G., \& Piñeros-Ventero, Y. L. (2018). Las notas de estados financieros en las normas internacionales de información financiera en el caso colombiano. Revista GEON (Gestión, Organizaciones Y Negocios), 5(1), 54-63. https://doi.org/10.22579/23463910.13

Bolaños-Bolaños, L. C. (2018). NIIF para pymes: relación costo beneficio del proceso de adopción y convergencia para las pymes en Colombia. IV Encuentro Nacional de Profesores de Contaduría Pública. Cali, Colombia: Universidad Cooperativa de Colombia sede Cali. https://n9.cl/izyk6 
Borrero, A. \& Ortiz, O. (2016). Pymes implementación de normas de información financiera internacional (grupo 2). Editorial Universidad del Norte. https://editorial.uninorte.edu.co/ gpd-pymes-implementacion-de-normas-de-informacion-financiera-internacional.html

Botero, A., Marulanda, C., Muñoz, L. \& Álvarez, M. (2018). Proceso de implementación de las NIIF en Colombia: un acercamiento a las autoridades de vigilancia definidas en la Ley 1314 de 2009. Contaduría Universidad De Antioquia, (73), 131-162. https://doi. org/10.17533/udea.rc.n73a06

Burgueño-Salas, E. (2020, 2 de noviembre). Colombia: número de hoteles y establecimientos similares 2005-2019. Statista. https://n9.cl/3n8pn

Chiriboga-Sánchez, G. (2013). Aplicación de la sección 23: ingresos de actividades ordinarias en una empresa pyme de turismo receptivo [disertación de grado, Pontificia Universidad Católica del Ecuador]. Repositorio PUCE. https:// n9.cl/k6a3

Del Franco Blanco, L., \& Gómez Lorduy, A. (2019). Contabilidad ambiental. Una reflexión en el marco de la ges- tión socialmente responsable de las empresas colombianas. Aglala, 10(2), 60-80 Recuperado de http://revistas. curnvirtual.edu.co/index.php/aglala/ article/view/1432

Departamento Administrativo Nacional de Estadística DANE (2020). Muestra Mensual de Hoteles (MMH) mayo 2019-2020. Boletín Técnico Muestra Mensual de Hoteles (MMH). https:// n9.cl/5o8xi

Departamento Administrativo Nacional de Estadística DANE (2021). El turismo en cifras: enero 2021. Informes de turismo. https://n9.cl/kvxb

Fierro-Martínez, A., M. y Fierro-Celis, F. (2015). Contabilidad General con enfoque NIIF para las pymes. Ecoe Ediciones.

Franco-Franco, W. (2018). Aspectos generales de las NIIF y el modelo de convergencia en Colombia [presentación de diapositivas]. Consejo Técnico de Contaduría Pública [CTCP]. https:// n9.cl/8ig4j

Fundación IASC (2009). Módulo 23: Ingresos de Actividades Ordinarias. Material de formación sobre NIIF para Pymes (versión 2010-2). https://n9.cl/bdea

Hoogervorst, H. (2015, 8 de julio). El costo histórico versus la medición del valor razonable. NIC-NIIF, Organización Interamericana de Ciencias Económicas [OICE]. https://n9.cl/i0539

IFRS Fundation (2016). CINIIF Interpretación 22: Transacciones en moneda extranjera y contraprestaciones anticipadas. IFRIC. https://n9.cl//4qv

Ministerio de Comercio, Industria y Turismo (2019). Estadísticas e informes enero - diciembre de 2019 [presentación de diapositivas]. MinCIT. https:// n9. cl/8w9i0 
Ministerio de Comercio, Industria y Turismo (2020). El turismo en cifras: julio 2020 [presentación de diapositivas]. MinCIT.https://n9.cl/g9w75

Moncayo, C. (2015, 30 de mayo). Reconocimiento contable de las ventas bajo NIIF. Instituto Nacional de Contadores Públicos [INCP]. https://n9.cl/1cev

NICNIIF. (s.f.). Qué es el IASB. Portal NICNIIF. https://www.nicniif.org/home/ iasb/que-es-el-iasb.html

Normas Internacionales de Información Financiera (2011, 25 de marzo). ¿Qué es el valor razonable? NIIF.CO. https:// n9.cl/ahx54

Organización Mundial del Turismo (2019, 21 de mayo). Turismo Internacional: aumentan cifras y la confianza. UNWTO. https://n9.cl/wozkk

Peña-Miranda, D., Guevara-Plaza, A., Fraiz-Brea, J., \& Botero, M. (2018). Prácticas de responsabilidad social empresarial en el sector hotelero. Estudio de casos en la ciudad de Santa Marta, Colombia. Cuadernos de Gestión 19 (1), 175-201. https://n9.cl/8rc8a

Registro Nacional de Turismo [RNT] (2021). Reporte de número de prestadores de servicios turísticos en Colombia. Estadísticas nacionales RNT Citur. https://n9.cl/a1v3n

Rodríguez-Aponte, J., Rodríguez-Aponte, I., Sánchez-Borrero, C., \& Franco-Rodríguez, D. (2015) Wild Guaviare una empresa ecoturística para el fomento y el desarrollo del deporte extremo en el municipio de San José del Guaviare. [trabajo de grado, Universidad de Los Llanos]. Repositorio Unillanos. https://bit.ly/2YZunPf

Rondi, G. R., Casal, M. C., Galante, M. J., Gómez, M. \& Giai Levra, V. (2020). Reconocimiento y medición de ingresos en la actividad hotelera. XL Jornadas Universitarias de Contabilidad (UUC), 1-10. http://nulan.mdp.edu. ar/3293/1/rondi-etal-2020.pdf

Salazar, E. E. (2011). Análisis de las implicaciones no financieras de la aplicación de la NIIF para PYME en las medianas entidades en Colombia. Cuadernos de Contabilidad, 12 (30), 211-241. Recuperado a partir de https://revistas.javeriana.edu.co/index.php/cuacont/ article/view/3116

T\&L Turism Leisure \& Sports \& Avia Export (2012). Diagnóstico de Naturaleza en Colombia. Plan de Negocio de Turismo de Naturaleza en Colombia. https://n9.cl/i1dpl

Vanegas, G. (2006). Ecoturismo, instrumento de desarrollo sostenible. Universidad de Antioquia. https://n9.cl/d7dx

Viajala. (2020, 22 de octubre). 5 hoteles ecológicos para hospedarse en Colombia. Viajala Blog. https://bit.ly/3zjGZNw 